\title{
Intelligent Mechanisms for Organising of Economic Activity of a Wagon Repair Depot
}

\author{
Nina Sirina \\ Urals State University of Railways, Mechanical Faculty, 66 Kolmogorova Str, Ekaterinburg, 620034, Russia \\ *Corresponding Author: Ninasirina@gmail.com
}

Copyright (c) 2013 Horizon Research Publishing All rights reserved.

\begin{abstract}
The paper presents the results of researches of intelligent mechanisms of the organizational system functioning and their developments to establish adaptive control system for plant manufacturing. Both a theoretical approach and practice of railway freight wagons plants are considered to create a control manufacturing system which is adaptive to changes of market conditions. The system of decision making support includes intelligent mechanisms of the plant manufacturing system functioning based on intelligent information systems as well as on learning, self-organizing expert mechanisms.
\end{abstract}

Keywords Organization, Intelligent, Control, Mechanism, Activity, Learning, Adaptation, Manufacture

\section{Introduction}

System science researches of organizational control include analysis and consideration of a human factor effect which should be understood as an activity of people or collectives (elements of a control system) [1-4]. It is caused by the existence of their own aims, not necessarily coinciding with the goal of the system in its entirety. Such active elements may utilize available information channels connected to the centre to improve a current or future state. To avoid information distortion passed by these elements to the centre of the system, it is necessary to consider the problem of information system design within a total problem of synthesis of such procedures as planning, regulation and stimulation accepted in the control mechanism. The theory as well as practice control for hierarchic organizations with a stochastic structure is connected to designing stochastic mechanisms of active system functioning (SFM) [2]. SFM includes an information subsystem and procedures of planning, regulating, and stimulating.

In practice, the very possibility for a complex organization control in dynamics with no complete information is based on intelligent information systems (IIS) [3]. IIS realize a model of identification and prediction and also form a foundation for control actions on the basis of current information, received from the active elements. Intelligent functioning mechanisms (IFM) include both SFM planning, regulating and stimulating procedures and IIS to identify the internal structure of active elements and their parameters, as well as to utilize internal elements' resources in accordance with the central goal. The main types of IFM are as follows: Learning functioning mechanisms (LFM), Self-organizing mechanisms (SOM), Expert intelligent mechanisms (EIM).

LFM provide a possibility of estimating of organizational potential parameters in their dynamics, supplying more information to plan the organization's output indices at the account of learning processes.

SOM combine learning and output indices planning (the way it is done in LFM) with the control of the organization's inputs, i.e. direct influence on the potential of an organization.

EIM combine learning with indistinct and qualitative commands from the centre and control based on those commands.

\section{Adaptive Mechanism for Organizing of Economic Activity of a Wagon Repair Depot}

Adaptive Mechanism for Organising of economic activity of a Wagon Repair Depot (AMO WRD) is considered as a system consisting of «strategic centre», «operative centre» and «executor» (Figure 1) [5, 6]. 


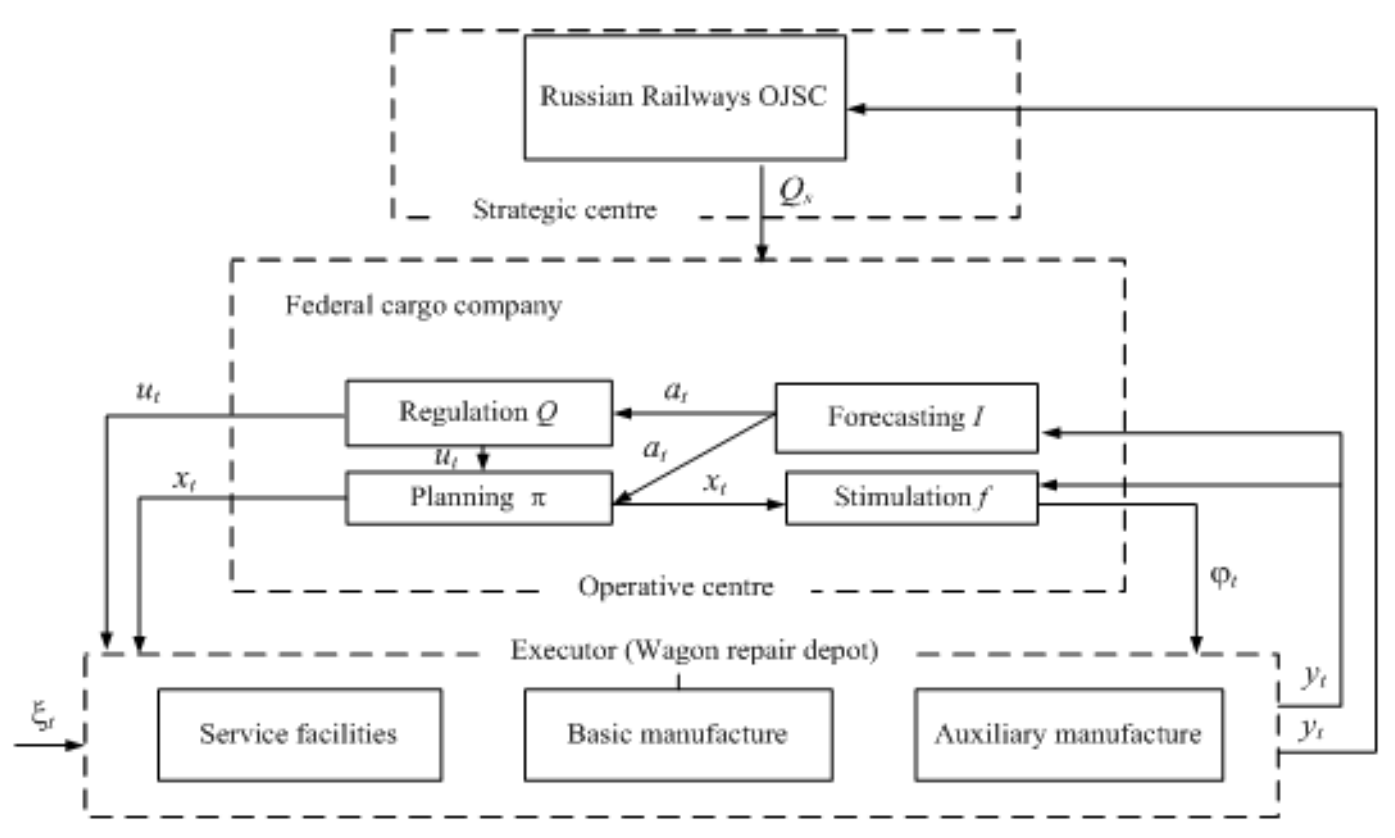

Figure 1. AMO WRD Structure

«Strategic centre», i.e. Russian Railways OJSC, regulates development of the whole complex on the basis of strategic forecasting and allocation of resources.

«Operative centre», i.e. Federal cargo company, possesses of the following functions: adaptive forecasting on the basis of learning (estimation), regulating of resources (resource), planning of tasks for repair (plan) and stimulating of «executor» (stimulus).

A wagon repair depot acts as «executor». It is supposed that its input is influenced by a stochastic hindrance and the result of its activity is characterised by an output. The quantity of outputs of each repair depot depends on its potential.

The system in the period $t, t=0,1 \ldots$ begins to function from the moment when Federal cargo company provides the input of the repair depot with operating influences such as plan and resource. Simultaneously the input is influenced by the environment in the form of a stochastic hindrance. The repair depot chooses an output. Federal cargo company observes the output, estimates it and extrapolates the estimate to the following period. Further, Federal cargo company defines resource and plan for the next period on the basis of this estimation using procedures of planning and regulating. On comparing of the actual output to the plan, the stimulus of the repair depot is defined. After that the system's functioning in period $t$ is over, and there comes period $t+1$, and so on.

The central goal of the repair depot during period $t$ conforms to its total discounted profit for all period of foresight. The repair depot chooses a current output with respect to maximising its central goal.

Synthesis AMO WRD forms the following types of mechanisms: Rigid regulation; Flexible response; Establishing of admissible activity limits. The rigid regulation mechanism provides equality of the output and plan at any capacity of the repair depot. For implementing of this mechanism, it is necessary and sufficient that the plan was certainly doable. The flexible response mechanism ensures complete use of the repair depot's capacity in each period; this is its priority. The flexible response mechanism is progressive as regards estimation if both the estimation and stimulus increase with growth of the output. The flexible response mechanism is regressive as regards estimation if maximum stimulus decreases with growth of the output estimation.

The priority of the mechanism of admissible activity limits establishing is co-ordination of the repair depot activity by the means of planning. The repair depot chooses an output equal to the plan if it is possible. If the plan proves impracticable because of any changes, the repair depot still tries its best to reach the planned target.

AMO WRD is put in operation at the enterprises of Sverdlovsk railway, a branch of Russian Railways OJSC.

A simple AMO WRD model applying just planning and stimulation procedures is used for a quantitative estimation of the repair depot output (Figure 2). On the basis of the current norm and output, an adaptive norm for the following period is defined (planning procedure). Comparison of the output to the norm provides a quantitative estimate (stimulation procedure).

Another elementary model of this mechanism is used for qualitative estimation of the output (rank) (Figure 3). It is calculated by association of the output with set $\Delta_{1}$ or $\Delta_{2}$ on the basis of ranging procedure. Ranging is connected with some losses: either it is output overestimating (erroneous placing of output $\Delta_{2}$ to $\Delta_{1}$ ) or output understating (erroneous placing of output $\Delta_{1}$ to $\Delta_{2}$ ). If classification of 
the output is correct, the loss is equal to zero, so it is necessary to adjust the procedure of ranging to minimise the loss.

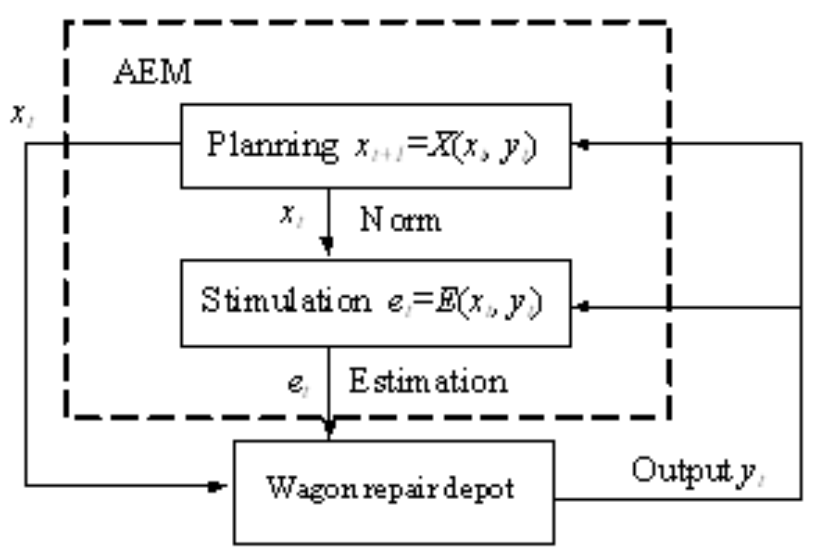

Figure 2. An adaptive estimating mechanism

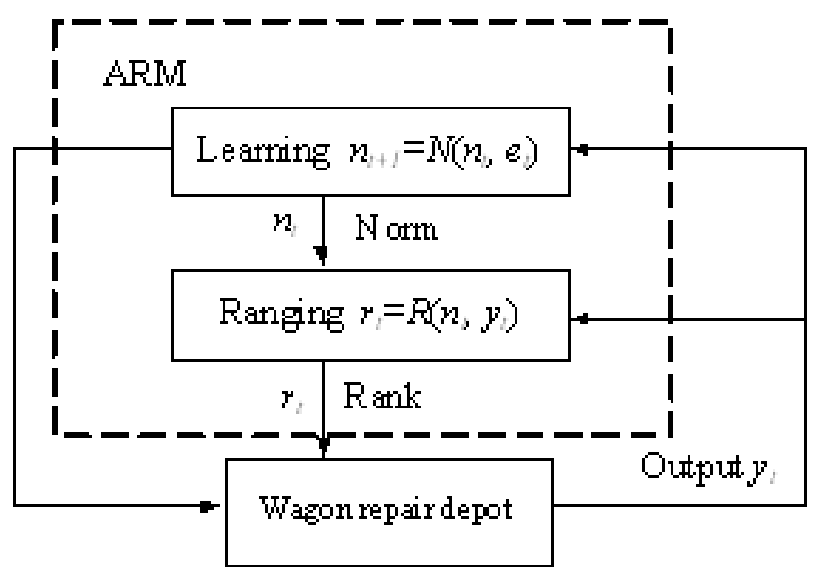

Figure 3. An adaptive ranging mechanism

For consecutive quantitative and qualitative estimation, an adaptive mechanism of estimation and ranging (AMER) is used (Figure 4). AMER represents a hierarchically ordered set of an adaptive estimating mechanism (AEM) and an adaptive ranging mechanism (ARM). In AEM an estimate is calculated under the formula:

$$
\begin{gathered}
e_{t}=f^{o}\left(x_{t}, y_{t}\right)=y_{t}-\chi\left(x_{t}, y_{t}\right) \\
\chi\left(x_{t}, y_{t}\right)=\left\{\begin{array}{l}
v\left(y_{t}-x_{t}\right), \text { if } x_{t} \leq y_{t} \\
\mu\left(x_{t}-y_{t}\right), \text { if } x_{t}>y_{t}
\end{array}\right.
\end{gathered}
$$

where $y_{t}-$ actual output in period $t ; x_{t}-$ norm of estimating in period $t$ calculated on the basis of an adaptive forecasting model of time numbers $x_{t}=x_{t-1}-\left(x_{t-1}-y_{t-1}\right) /(t-1), \quad ; \quad \chi\left(x_{t}, y_{t}\right)$ stimulation function for a deviation of $y_{t}$ from the norm; $v$ - encouragement coefficient for over fulfillment of the norm; $\mu-$ punishment coefficient for failure of the norm, $v, \mu>0$.

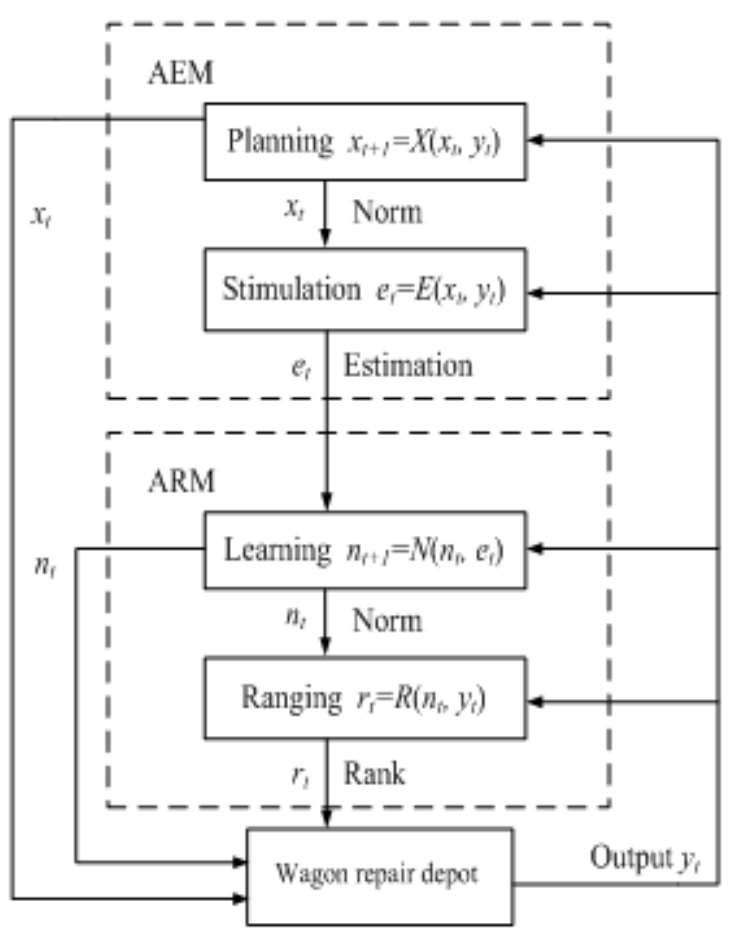

Figure 4. An adaptive mechanism of estimation and ranging

ARM carries out its function with the aid of norms calculated by means of stochastic procedure of images recognition, by approximation of results of learning with a teacher:

$$
\begin{aligned}
& n_{t+1}^{i}=N_{t}^{i}\left(n_{t}^{i}, e_{t}\right)= \\
& =n_{t}^{i}-\gamma_{t}\left(n_{t}^{i}-\frac{\omega_{12}^{i}-\left(\omega_{12}^{i}+\omega_{21}^{i}\right) S_{i}\left(e_{t}\right)-h}{l}\right)
\end{aligned}
$$

where $i$ - norm of ranging number, $i=1,3, n_{t}^{1}-2^{\text {nd }}$ rank norm of ranging; $\omega_{12}^{l}-$ size of losses in case of erroneous association of $2^{\text {nd }}$ rank with $1^{\text {st}} ; \omega_{21}^{l}-$ size of losses in case of erroneous association of $1^{\text {st }}$ rank with $2^{\text {nd }} ; n_{t}^{2}-3^{\text {rd }}$ rank norm of ranging; $\omega_{23}^{2}-$ size of losses in case of erroneous association of $3^{\text {rd }}$ rank with $2^{\text {nd }} ; \omega_{32}^{2}-$ size of losses in case of erroneous association of $2^{\text {nd }}$ rank with $3^{\text {rd }} ; x_{t}^{3}-4^{\text {th }}$ rank norm of ranging; $\omega_{34}^{3}$ - size of losses in case of erroneous association of $4^{\text {th }}$ rank with $3^{\text {rd }} ; \omega_{43}^{3}-$ size of losses in case of erroneous association of $3^{\text {rd }}$ rank with $4^{\text {th }}$;

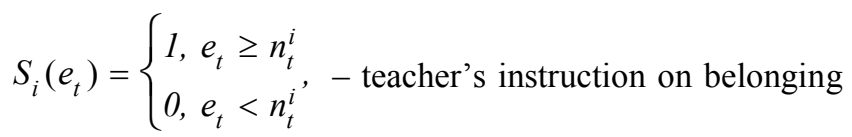
of the estimate, $\gamma$ - adaptation step, $t=1, T, T$ - number 
of considered periods.

\section{Integrated Estimation of a Wagon Repair Depot}

Integrated estimation of a wagon repair depot (IOWRD) serves for carrying out both retrospective and perspective analysis. It is made on the basis of AMER [7,8].

A technique of IOWRD formation is presented in this paper. It is worked out on the basis of Balanced Scorecard (BSC) system general approach. At first, primary indicators and their standard values are selected. These indicators are further grouped according to the depot's spheres of activity. In each group a local estimate and rank are formed for a certain sphere of activity. Local ranks are united into intermediate ranks by means of a convolution matrix which is a table function.

The scheme of forming of an integrated estimate of a wagon repair depot is shown in Figure 5. Indicators of the depot's activity fall into three groups: industrial, financial and personnel. Local estimations are defined by means of AMER.

Local estimation of industrial activity is influenced by the output and coefficient of quality. The output is estimated on the basis of achievement of planned targets on repair of freight cars belonging to Russian Railways OJSC and to other industrial enterprises (taking into account quantity and types of the repaired wagons and spare parts). The coefficient of quality shows efficiency of scientific and technical achievements and best practices used at repair as well as existence of defects. Local estimates and ranks as regards quantity of the output are received by means of AMER of released production and as regards its quality with the aid of AMER of quality of repair.

Local estimation of personnel selection is defined by their conformity to the nomenclature of professions and by safety coefficient which describes labour discipline (quantity of dismissals, penalties, awards, etc.), attitude to work and psychological atmosphere at the enterprise. This kind of local estimation is received by means of AMER of safety and AMER of personnel.

Local estimation of financial activity is determined by profit and profitability of the repair depot. A local estimate on profit is received by means of the rational adaptive mechanism of estimation and profit ranging. A local estimate on profitability is received by means of AMER of profitability.

Intermediate ranks of industrial and financial activity as well as that of personnel are formed by means of convolution matrixes (respectively 1, 2 and 3 in Figure 5).

Further an estimate of economic activity is defined through uniting the local ranks in industrial and personnel areas (convolution matrix 4 in Figure 5). Forming of an integrated estimate is carried out with the help of convolution matrix 5 (Figure 5).

Procedures of IOWRD are hierarchically ordered so that a decision maker can analyse both final and intermediate results of economic activity of the depot down to initial data. It is possible to estimate a whole enterprise, a certain site and a worker. 


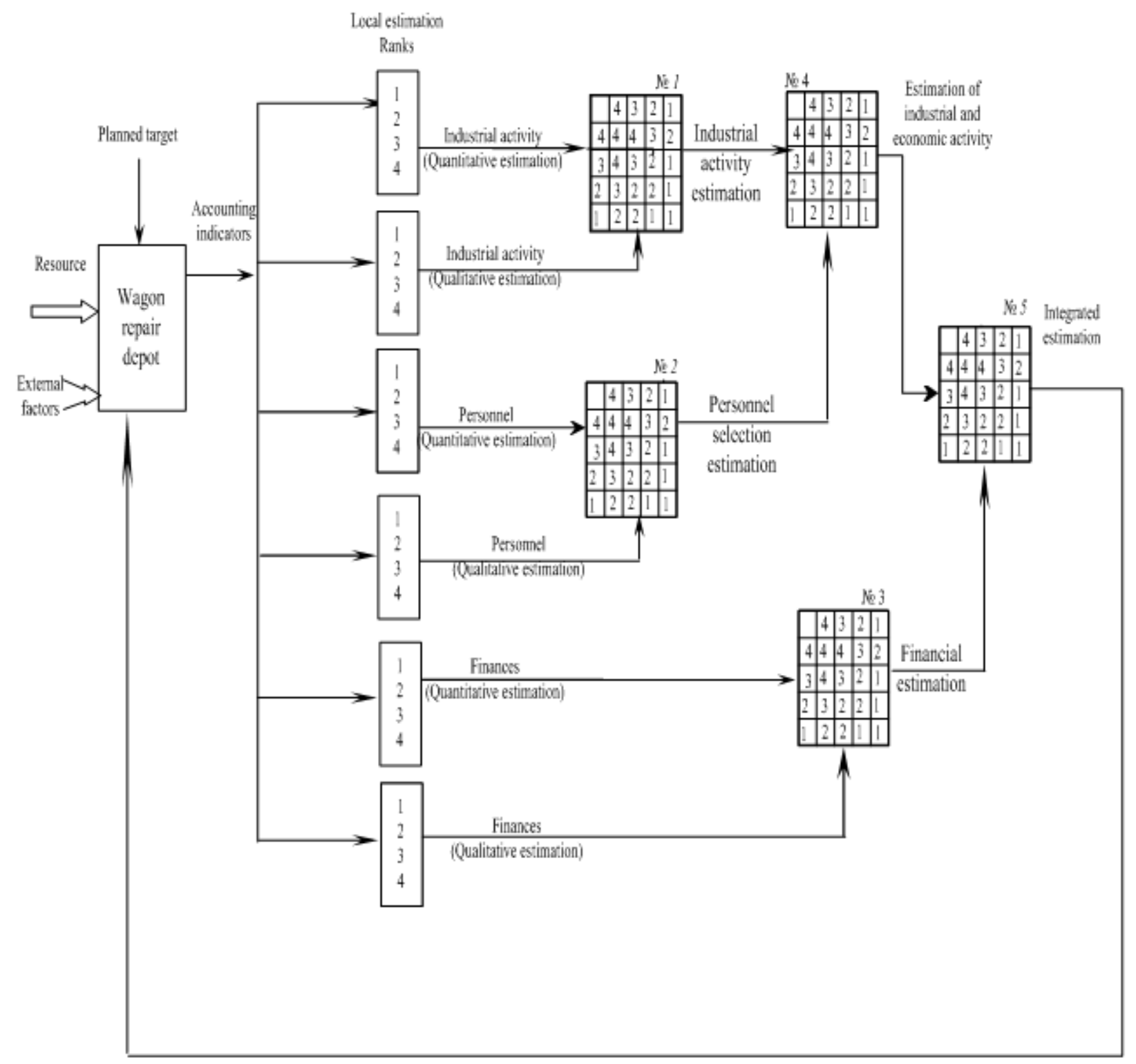

Figure 5. Scheme of IOWRD formation

\section{An Example of Using Adaptive Mechanism of Estimation And Ranging}

The process and results of functioning the adaptive mechanism of estimation and ranging has been shown on the basis of the control of fulfillment of the wagon repair plan.

The software for formation of norms for fulfillment of the plan for depot repair of private wagons, estimation, standards and ranging has been realized in Microsoft Excel.

In AOM on the basis of analyzing the fulfillment of the plan for depot wagon repair $y_{t}$ adaptive estimation standard for fulfillment of the depot wagon repair for the future period $t+1 \quad x_{t+1}^{o}=x_{t}^{o}-\left(x_{t}^{o}-y_{t}\right) / t, t=1,2, \ldots .52 ; \quad x_{0}^{o}=x^{o} \quad$ (Table $1)$ is formed. The estimation norm for the period $\mathrm{t}\left(x_{t}^{o}=x \mathrm{t}\right)$ is corrected considering the fulfillment of the depot wagon repair within the past period (Figure 6).

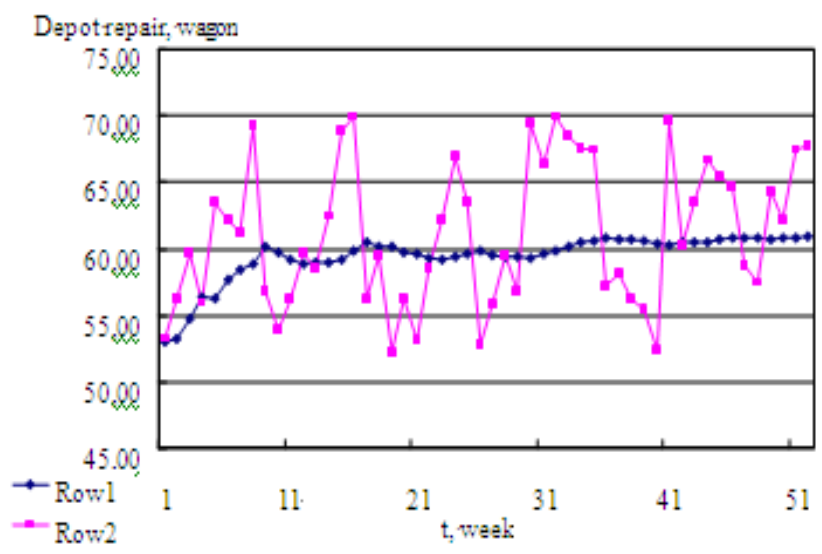

Figure 6. Diagram of fulfillment of depot wagon repair and the norm for its estimation 
Table 1. Value of fulfillment of depot wagon repair and its norm

\begin{tabular}{|c|c|c|c|c|}
\hline $\begin{array}{c}\text { Period } \\
\mathrm{N}\end{array}$ & $\begin{array}{c}\text { Fulfillmen }_{t} \\
\text { of depot } \\
\text { wagon } \\
\text { repair }\end{array}$ & $\begin{array}{c}\text { Adaptive } \\
\text { norm, } \\
x_{t}^{o}\end{array}$ & $\begin{array}{c}\text { Quantitative } \\
\text { estimation } \\
n_{t} \\
\end{array}$ & $\begin{array}{c}\text { Qualitative } \\
\text { estimation } \\
e_{t}^{o} \text { rank }\end{array}$ \\
\hline 0 & & 53,00 & & \\
\hline 1 & 53,25 & 53,00 & 0,05 & 3 \\
\hline 2 & 56,25 & 53,25 & 0,60 & 3 \\
\hline 3 & 59,58 & 54,75 & 0,97 & 3 \\
\hline 4 & 55,89 & 56,36 & $-0,09$ & 2 \\
\hline 5 & 63,41 & 56,24 & 1,43 & 3 \\
\hline 6 & 62,12 & 57,68 & 0,89 & 3 \\
\hline 7 & 61,24 & 58,42 & 0,56 & 3 \\
\hline 8 & 69,25 & 58,82 & 2,09 & 4 \\
\hline 9 & 56,78 & 60,12 & $-0,67$ & 2 \\
\hline 10 & 53,85 & 59,75 & $-1,18$ & 2 \\
\hline 11 & 56,12 & 59,16 & $-0,61$ & 2 \\
\hline 12 & 59,67 & 58,89 & 0,16 & 3 \\
\hline 13 & 58,59 & 58,95 & $-0,07$ & 2 \\
\hline 14 & 62,47 & 58,92 & 0,71 & 3 \\
\hline 15 & 68,79 & 59,18 & 1,92 & 3 \\
\hline 16 & 69,96 & 59,82 & 2,03 & 4 \\
\hline 17 & 56,24 & 60,45 & $-0,84$ & 2 \\
\hline 18 & 59,51 & 60,20 & $-0,14$ & 2 \\
\hline 19 & 52,21 & 60,17 & $-1,59$ & 2 \\
\hline 20 & 56,21 & 59,75 & $-0,71$ & 2 \\
\hline 21 & 53,14 & 59,57 & $-1,29$ & 2 \\
\hline 22 & 58,59 & 59,26 & $-0,13$ & 2 \\
\hline 23 & 62,21 & 59,23 & 0,60 & 3 \\
\hline 24 & 66,87 & 59,36 & 1,50 & 3 \\
\hline 25 & 63,49 & 59,68 & 0,76 & 3 \\
\hline 26 & 52,75 & 59,83 & $-1,42$ & 2 \\
\hline 27 & 55,86 & 59,56 & $-0,74$ & 2 \\
\hline 28 & 59,48 & 59,42 & 0,01 & 3 \\
\hline 29 & 56,78 & 59,42 & $-0,53$ & 2 \\
\hline 30 & 69,42 & 59,33 & 2,02 & 4 \\
\hline 31 & 66,32 & 59,67 & 1,33 & 3 \\
\hline 32 & 69,87 & 59,88 & 2,00 & 4 \\
\hline 33 & 68,43 & 60,19 & 1,65 & 3 \\
\hline 34 & 67,49 & 60,44 & 1,41 & 3 \\
\hline 35 & 67,42 & 60,65 & 1,35 & 3 \\
\hline 36 & 57,26 & 60,84 & $-0,72$ & 2 \\
\hline 37 & 58,12 & 60,74 & $-0,52$ & 2 \\
\hline 38 & 56,27 & 60,67 & $-0,88$ & 2 \\
\hline 39 & 55,36 & 60,56 & $-1,04$ & 2 \\
\hline 40 & 52,38 & 60,42 & $-1,61$ & 1 \\
\hline 41 & 69,60 & 60,22 & 1,88 & 4 \\
\hline 42 & 60,28 & 60,45 & $-0,03$ & 2 \\
\hline
\end{tabular}

\begin{tabular}{|l|l|l|l|l|}
\hline 43 & 63,53 & 60,45 & 0,62 & 3 \\
\hline 44 & 66,66 & 60,52 & 1,23 & 3 \\
\hline 45 & 65,52 & 60,66 & 0,97 & 3 \\
\hline 46 & 64,58 & 60,77 & 0,76 & 3 \\
\hline 47 & 58,72 & 60,85 & $-0,43$ & 2 \\
\hline 48 & 57,48 & 60,80 & $-0,66$ & 2 \\
\hline 49 & 64,24 & 60,73 & 0,70 & 3 \\
\hline 50 & 62,27 & 60,81 & 0,29 & 3 \\
\hline 51 & 67,46 & 60,84 & 1,32 & 3 \\
\hline 52 & 67,75 & 60,97 & 1,36 & 3 \\
\hline
\end{tabular}

Fig 6 Row 2 shows fulfillment of depot wagon repair and row 1 - the norm for estimation.

Further, the procedure of estimation of fulfillment of depot wagon repair is formed. The procedure is based on correlation of the norm and real fulfillment of depot repair of private wagons.

On the basis of quantitative estimation $n_{t}$ the ranging norm $x_{i t+1}^{P}$ is formed in the ranging mechanism $x_{i t+1}^{P}$.

The diagram of estimation of fulfillment of depot wagon repair is shown in Figure.7.

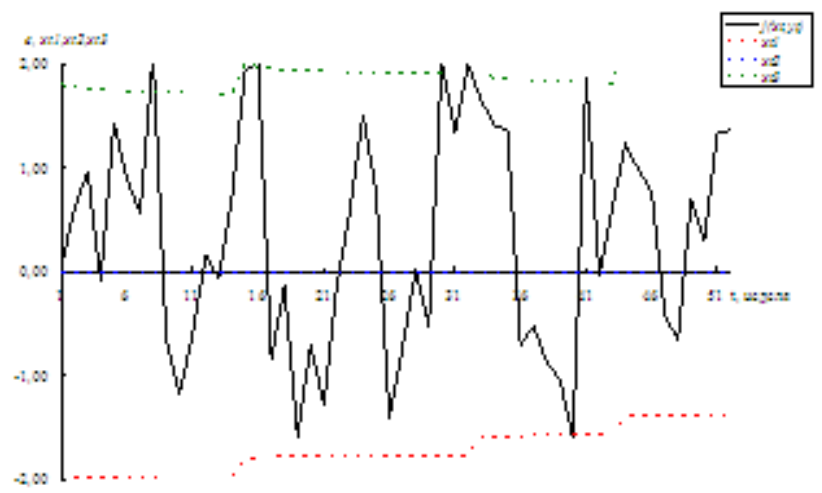

Figure 7. Diagram of estimation and norms for estimation ranging of fulfillment of depot repair

The estimation rank for fulfillment of depot wagon repair is further formed according to the 4-point system.

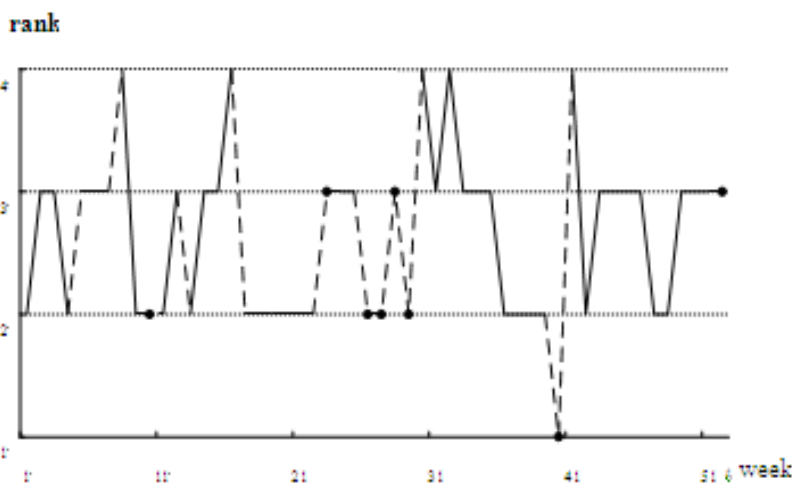

Figure 8. Diagram of estimation ranks for fulfillment of depot wagon repair 


\section{Conclusion}

Adaptive planning for a wagon repair enterprise provides continuous control and regulation of manufacturing process. Moreover, evaluation of output of manufacturing areas and enterprise as a whole is made. On the basis of estimation the need for workforce by qualifications is clarified as well as supplies with materials, tools and devices. Prevention of deviations from the given working schedule is secured, planning and efficiency are provided. The ranks of output results are used to encourage the staff.

\section{REFERENCES}

[1] D. Borodin, I. Gurlev., A. Klukvin., V. Tsyganov., Adaptive Mechanism for Sustainable Development, Systems Science, Vol. 30 (2004), No. 2, pp.89-95.

[2] V. Burkov, V. Tsyganov, Stochastic mechanisms of the active system functioning, Preprints of the 2nd IFAC Symposium on
Stochastic Control, Moscow 1986, Vol.1, pp. 259-263.

[3] V. Burkov, V. Tsuganov, Adaptive information system to control organization activity, Preprints of IFIP Conference on Governmental and Municipal Information Systems, Budapest 1987, pp. 85-95.

[4] V. Tsyganov, Progressive Adaptive Mechanisms for the International Cooperation, Preprints of the 17th IFAC World Congress, Seoul 2008, pp. 6697-6702.

[5] N.F. Sirina. Organization of railway cars' repair on the adaptive mechanisms, Transport Urala. Vol. 19 (2008), No. 4, pp. 10-14.

[6] N.F. Sirina. The principles of mechanisms' design of railway sector, Transport Urala. Vol. 13 (2007), No. 2, pp. 62-68.

[7] N.F. Sirina. Modelling ofcfr-repair complex management, Herald of the Ural State University of Railway Transport. Vol. 1 (2009), No. 1, pp. 12-20.

[8] N.F. Sirina, F.M. Simonov. Evaluation of competitiveness of car repair enterprise, Herald of the Ural State University of Railway Transport. Vol. 11 (2011), No. 3, pp. 66-74. 